\begin{tabular}{|c|c|}
\hline & $\begin{array}{l}\text { International Journal of Trend in Scientific } \\
\text { Research and Development (IJTSRD) }\end{array}$ \\
\hline 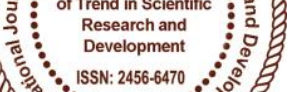 & International Open Access Journal \\
\hline 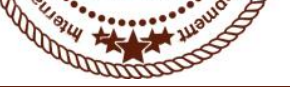 & ISSN No: $2456-6470 \mid$ www.ijtsrd.com | Volume - 2 | Issue -3 \\
\hline
\end{tabular}

\title{
Scaring Animals using Assembler Language
}

\author{
${ }^{1}$ Pavithra. M. H, ${ }^{2}$ Nivetha. R, ${ }^{3}$ Haritha. M, ${ }^{4}$ Sandra Karunya, ${ }^{5}$ Madhan Gopi \\ ${ }^{1,2,3}$ Students, ${ }^{4,5}$ Proffessors \\ Computer Science and Engineering, Sri Muthukumaran Institute of Technology \\ Chennai, Tamil Nadu, India
}

\begin{abstract}
Human-Wild animal conflict is the major problem in the forest borders with large wild animal herds. Human conflicts with wild animals often occur, along with the narrowing of the wild animal habitat due to industrial and residential interests. The negative impacts of human wild animal conflict is on a large scale slaughter of wild animals. This problem which leads to crop damage, human death and injuries caused by wild animals, and wild animals being killed by humans. Wild animal Intrusion has been on the rise in the forest border areas with herds of wild animals straying into human habitation and creating a great loss to their properties. The surveillance and tracking of wild animals are difficult due to their size and nature of movement. Prevention system for indications of human wild animal conflict is absolutely necessary. So an intelligent electronics system is necessary which can be affixed to avoid the human-wild animal conflicts. In this paper, an automated system to detect the intrusion of wild animals into the human habitat in forest borders is proposed. Basic idea behind this work is to generate the sound signal which can be inaudible to human and irritating for wild animal. Such instrument can be mounted on forest borders so that wild animal herds will go back into the forest. The proposed is very efficient with good computation power and low cost.
\end{abstract}

\section{INTRODUCTION}

Indian sub-continent is diverse in biological and nonbiological aspects. The diversity in the flora of the forest areas in the Country attracts a wide range of animals to have it as the habitat. One of the most prominent species in the Indian forest is the elephant. India is the major habitat for 60 percent of the Asian elephants (Elephants Maximum) of the total estimated population of 40,000-50,000. Asian elephants lose their habitat as the forest areas are rehabilitated to human settlements, industries and agricultural lands. These conversions lead to the increased shortage of the natural food and water resources needed by the herd of elephants. Shortage and fragmentation of habitat makes the herd of wild elephants to enter into the human habitat in the forest borders and roadways. There exists a severe damage to crops, human lives and also the elephants.

The crop damage is costlier as the herd damages the larger portion of the vegetation when it moves into the farm lands. This loss by elephants can be mitigated by taking precaution measures on intrusion of the herd. But the detection and tracking of the herds is hard due to their size and complex nature of the movement. The elephants are considered endangered species as it faced heavy loss of lives by poachers.

The poaching for ivory of the elephants, attacks made by humans to protect their farm lands, captivation of baby elephants and accidents are some common reasons of the reduction of elephant population.

\section{II.EXISTING SYSTEM:}

The conflict between humans and animals is seen across the country in a variety of forms, including monkey menace in the urban areas, crop raiding by wild pigs and so on. Providing effective solutions for human-animals conflicts now one of the most significant challenges all over the world. In this paper, a wireless sensor network based on UWB technology is used to deploy intrusion detection. By analyzing the 
characteristics of Ultra wide band (UWB) signals, convolution alneural network is used to learn the characteristics of UWB signals automatically. And finally the SVM or Softmax classifier is used to classify human beings from animals. Several experiments are tested in corn field and the experimental results show that the method proposed in this paper can detect human and animal intrusion very effectively and improve the accuracy of detection by nearly $16 \%$ compared to the traditional manual extraction.

\section{III.PROPOSED WORK:}

\section{a. Block Diagram:}

The proposed method includes three dominant phases fore-recognition and classification of animals, namely: pre-processing, feature extraction and classification. In the pre-processing step, animal image is resized and segmented by elimination of background noises through the similarity based region merging technique with object of our interest. This results in obtaining image with texture features of the corresponding animal in it.

Later, the pre-processed image containing the texture features is considered for extraction of different properties with the inclusion of varying scales and orientations. With the usage of gabor filters in the gabor feature extraction technique, a feature vector for each animal image is generated.

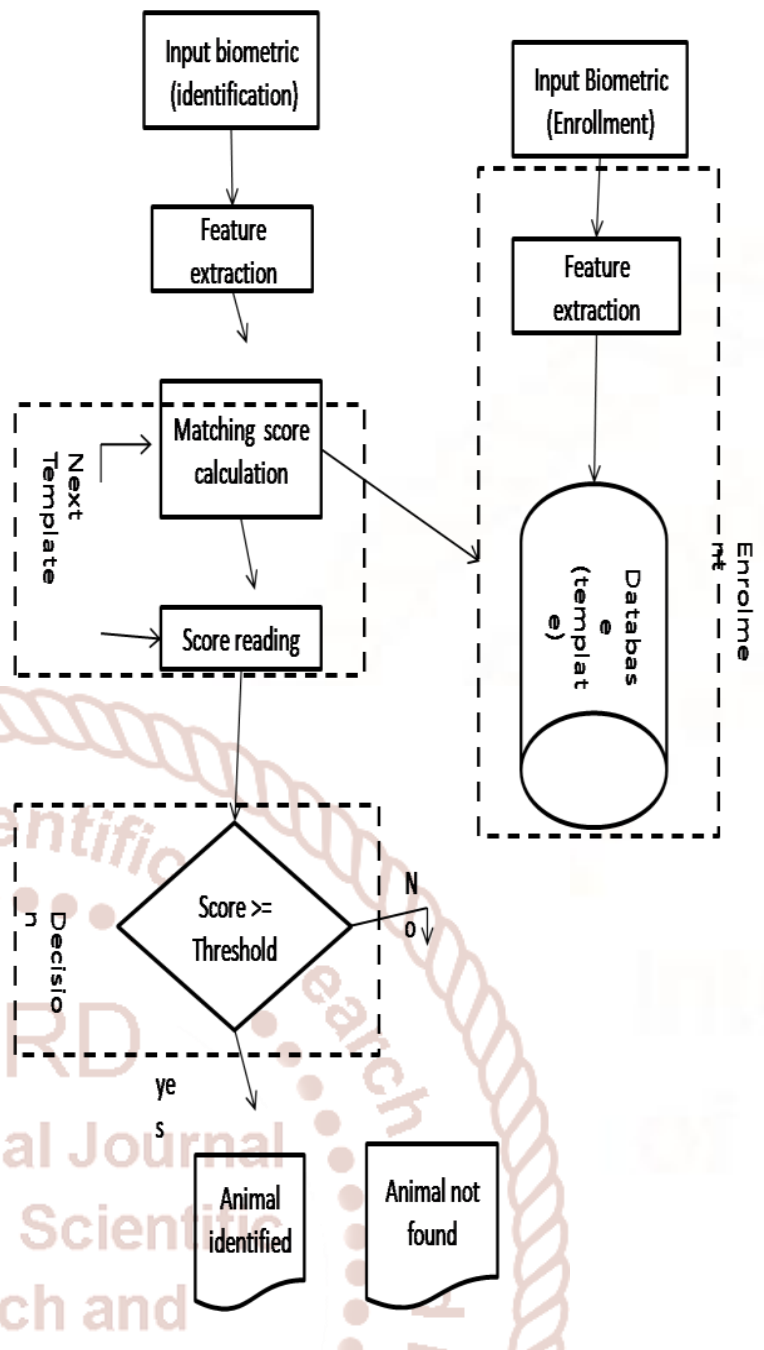

Figure 1. Block diagram for Implementation

Various Modules involved for this purpose are:

- Camera Interface

- Animal Detection

- Animal Recognition

- Disturbing tone generation

Hence, the resultant feature matrix obtained by combining all the feature vectors is fed to the Neural training for the purpose of classification of animals. Classification accuracy is measured by considering several ratios of training and testing samples with respect to the matched labels in the feature matrix. Performance measurements are made with respect to time through parallel computation which are applied for major processing tasks.

\section{b. Segmentation}

The segmentation of animal image results in background elimination to obtain the image containing texture features. The segmentation is carried out by similarity based region merging 
technique. In this technique we need to indicate the location and region of object and background by using strokes which are called markers for the removal of background noises in the image. This results in a segmented image that leaves texture features which forms a major factor for the extraction of features.

\section{c. Feature Extraction}

The pre-processed images are subjected for the extraction of features. We have used gabor features that are obtained from gabor filter responses which includes statistical calculations that can be performed on the resulting feature matrix of the image dataset like energy calculation, homogeneity level and similarity from the texture features. Before feature extraction takes place, some similarity criterion has to be defined that are normally specified in terms of set of feature-measures, which provide a quantitative measure of certain texture characteristics. These feature measures are alternatively referred to as texture-measures or features.

Groups of feature measures that are assembled for classification purposes are often referred as featurevectors. We have used Gabor features primarily based on Gabor filter responses for the given input image. The responses over the input images are calculated for a set of filters, forming a filter bank tuned to various orientations and frequencies. In the proposed methods, the principles are briefly analyzed and the novel methods for selecting the filter parameters are also devised.

\section{HARDWARE AND SOFTWARE REQUIREMENTS}

The hardware requirements that are necessary for implementing Scaring animals using machine language project includes are as follows:

- HQ Video Cam: HQ is a High Quality camera. Although it has less quality compared to HDR(High Dynamic Quality), it is more efficient and cost effective for small scale purposes.

- Arduino Mega: It is a microcontroller board based on ATmega1280(datasheet). It has 54 digital input/output pins(of which 14 can be used as PWM outputs), 16 analog inputs, 4 UARTs (hardware serial ports), a $16 \mathrm{MHz}$ crystal oscillator, a USB connection, a power jack, an ICSP header and a reset button.

- Multi-tone Buzzer: Buzzer or beeper is an audio signalling device which may be mechanical, electro-mechanical or piezo-electric. Here, this buzzer has been characterized by more than one musical tone. This multi-tone are used to scare animals from entering various restricted places where it has to be surveyed and protected from animals.

The various software's that the project requires are as follows:

\section{- MATLAB:}

Matrix Laboratory is a multi-paradigm numerical computing environment. Although MATLAB is intended primarily for numerical computing, an optional toolbox uses the MuPAD symbolic engine, allowing access to symbolic computing abilities.

\section{- Image Processing Toolbox:}

It provides a comprehensive set of reference-standard algorithms and workflow apps for image processing, analysis, visualization, and algorithm development. It can a perform image segmentation, image enhancement, noise reduction, geometric transformations, image registration, and 3D image processing.

Image Processing Toolbox apps automate common image processing workflows.. It is capable of interactively segment image data, compare image registration techniques, and batch-process large data sets. Visualization functions and apps explore images, 3D volumes, and videos, adjust contrast, create histograms and manipulate regions of interest(ROIs).

\section{ADVANTAGES:}

- Scaring animals are on a good intention that the wild animals being entering villages and damaging the crops on an large scale can be avoided.

- Prevents human hunting animals to protect themselves from being attacked. 


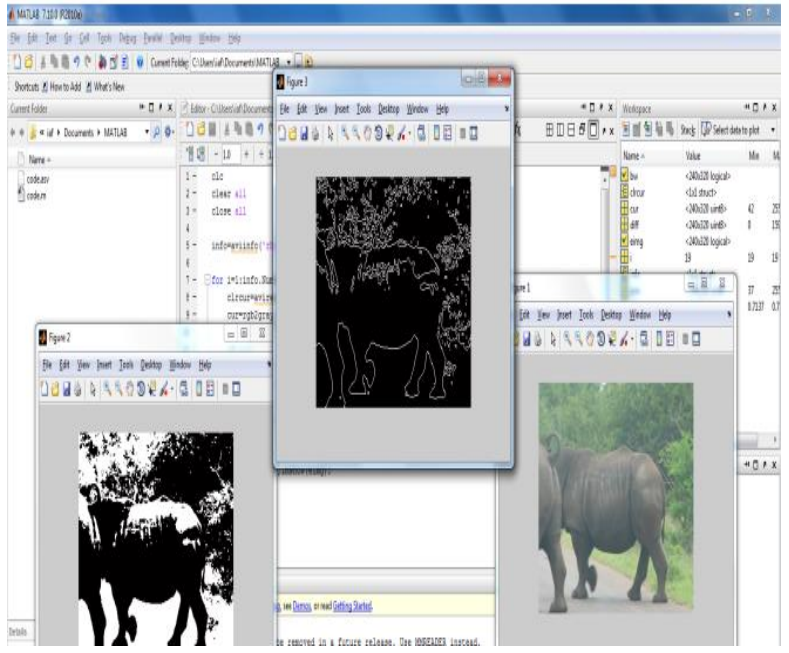

Figure 2. Implementation of Animal detection using MATLAB

\section{CONCLUSION:}

A neural training with back propagation algorithm is utilized to recognize various animals based on shape and texture and color. Neural training will be done with different views (front, rear and side view) of wild animals color, shape and pattern features. A live camera feed will be analyzed through MATLAB video processing algorithm to extract the image features and will be compared with real time neural trained database. Database will be constructed with wild animal image features along with their disturbing frequency range. The similarity result of the database with camera feed will trigger the speaker with stored frequency level.

\section{FUTURE WORK:}

The neural trained work will only recognize trained animals, it can be further trained to recognize other type of Human intrusions too. Those intrusion of humans can be used in the military areas where the border security can be increased. The opponent or enemy country people can be therefore detected through this identification of human intrusion.

\section{REFERENCES:}

1) $\mathrm{Y} H$ Sharath Kumar, Manohar $N$, Chethan $H$ K(2014). Animal Classification System: A block based Approach. ICICT 2014.

2) Mikolajczyk, Krystian, and CordeliaSchmid. "A performance evaluation of local descriptors."
Pattern Analysis and Machine Intelligence,IEEE Transactions on 27.10 (2005): 1615-1630.

3) Felzenszwalb, P. F., Girshick, R. B., McAllester, D., \&Ramanan, D.(2010). Object detection with discriminatively trained part-basedmodels. Pattern Analysis and Machine Intelligence, IEEE Transactionson, 32(9), 1627-1645.

4) Berg, Alexander C., Tamara L. Berg, and Jitendra Malik. "Shapematching and object recognition using low distortioncorrespondences."Computer Vision and Pattern Recognition, 2005. CVPR2005. IEEE Computer Society Conference on.Vol. 1.IEEE, 2005.

5) Krijger, Hans, Greg Foster, and Shaun Bangay. "Designing a Framework for Animal Identification." (2008).

6) Haralick, Robert M., KarthikeyanShanmugam, and Its' HakDinstein. "Textural features for image classification." Systems, Man and Cybernetics, IEEE Transactions on 6 (1973): 610-621.

7) Dasarathy, Belur V. "Nearest neighbor ( $\{\mathrm{NN}\})$ norms: $\{\mathrm{NN}\}$ pattern classification techniques." (1991).

8) Yuan, Xiao-Tong, Xiaobai Liu, and Shuicheng Yan. C"Visual classification with multitask joint sparse representation." Image Processing, IEEE Transactions on 21.10 (2012): 4349-4360.

9) TiloBurghardt. "Real time face detection and tracking of animals" (2006).. In: conference proceedings of Neural Network Applications in Electrical Engineering

10) Cristianini, Nello, and John Shawe-Taylor. An introduction to support vector machines and other kernel-based learning methods. Cambridge university press, 2000.

11) Ning, Jifeng, et al. "Interactive image segmentation by maximal similarity based region merging." Pattern Recognition 43.2 (2010): 445456.

12) $\mathrm{Yu}, \mathrm{Xiaoyuan}$, et al. "Automated identification of animal species in camera trap images." EURASIP Journal on Image and Video Processing 2013.1 (2013): 1-10.

13) Zeppelzauer, Matthias. "Automated detection of elephants in wildlife video."EURASIP journal on image and video processing 2013.1 (2013): 1-23. 
14) Narayanaswami, Ranga, et al. "Investigation of kinematic features for dismount detection and tracking." SPIE Defense, Security, and Sensing. International Society for Optics and Photonics, 2012.

15) Afkham, HeydarMaboudi, et al. "Joint visual vocabulary for animal classification." Pattern Recognition, 2008.ICPR 2008. 19th International Conference on.IEEE, 2008.

16) Ma, Lia, Yunhong Wang, and Tieniu Tan. "Iris recognition based on multichannel Gabor filtering." Proc. Fifth Asian Conf. Computer Vision. Vol. 1. 2002.

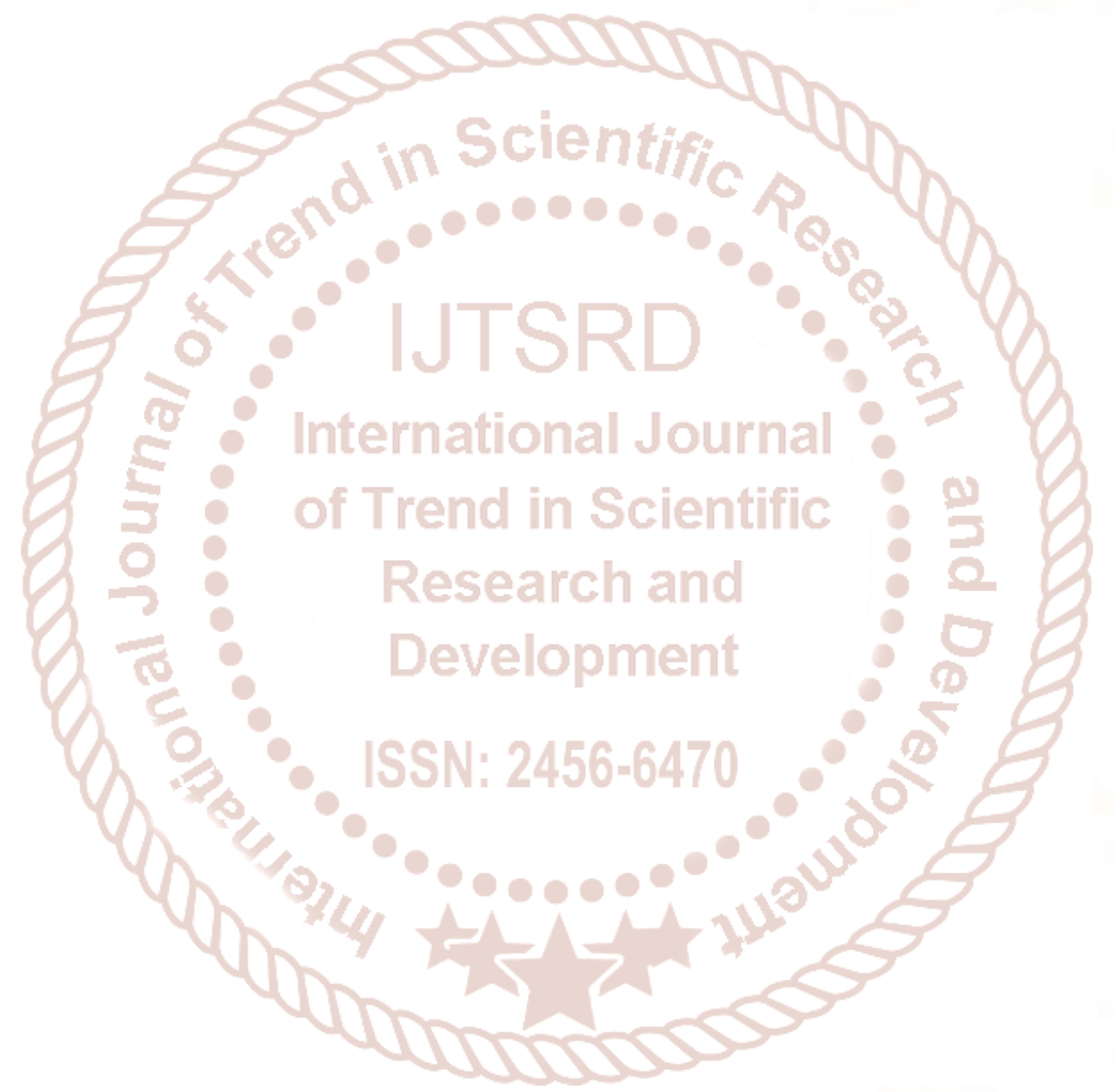

